\title{
An Extensive Extraperitoneal Insufflation due to Veress Needle Associated Complication
}

\author{
${ }^{1}$ Phornsawan Wasinghon, ${ }^{2}$ Kuan-Gen Huang
}

\section{ABSTRACT}

Aim: The case report presented of the uncommon extensive extraperitoneal insufflation due to Veress needle (VN) procedure of laparoscopy.

Background: The minimally invasive surgery has become the method of choice for the most benign disease. Creation of pneumoperitoneum is the first step of a laparoscopy. The Veress needle is placed blindly into the abdomen. There are major and minor complications of laparoscopy. The purpose of this study was to expose the minor complication outcome of extraperitoneal insufflation with $\mathrm{VN}$ access. The complication of this operation occurred during the laparoscopic surgical staging of endometrial cancer.

Case description: A 61-year-old woman, para two of vaginal deliveries, had previous laparoscopic Gilliam uterine suspension owing to uterine prolapse. She had menorrhagia for 6 months after her menopausal age of 53 years. The uterine had undergone curettage, and the histopathology displayed endometrial carcinoma. The treatment was laparoscopic surgical staging. While the $\mathrm{VN}$ was inserted and $\mathrm{CO}_{2}$ insufflation was taking place with $15 \mathrm{~mm} \mathrm{Hg}$, a minor intraoperative complication had occurred. The laparoscopy revealed Gilliam uterine suspension (GUS) where the extraperitoneal emphysema had occurred. The extraperitoneal emphysema was released with a needle gauge No.18 exteriorly during surgery.

Conclusion: The extensive extraperitoneal insufflation image shows a minor complication that is very uncommon reviewed and can thus be educational for the endoscopist.

Clinical significance: The VN was inserted vertically following the creation of pneumoperitoneum at a pressure of 10 to $15 \mathrm{~mm}$ $\mathrm{Hg}$, the Veress needle was removed. Then a $10 \mathrm{~mm}$ disposable shielded trocar was introduced in the pelvic cavity.

Keywords: Extraperitoneal insufflation, Gilliam uterine suspension, Laparoscopic complication, Veress needle.

How to cite this article: Wasinghon P, Huang K. An Extensive Extraperitoneal Insufflation due to Veress Needle Associated Complication. J South Asian Feder Obst Gynae 2018;10(3):222224.

${ }^{1}$ Gynecologic Oncology ${ }^{2}$ Associate Professor

${ }^{1}$ Department of Obstetrics and Gynecology, Buddhachinnaraj Hospital and Naresuan University,Phitsanulok, Thailand

${ }^{2}$ Department of Obstetrics and Gynecology, Chang Gung Memorial Hospital at Linkou, Kweishan, Taoyuan, Taiwan

Corresponding Author: Kuan-Gen Huang, Associate Professor, Chang Gung Memorial Hospital, Department of Obstetrics and Gynecology, Taoyuan, Linkou, Taiwan, e-mail: kghuang@ms57. hinet.net
Source of support: Nil

Conflict of interest: None

Date of received: $07 / 16 / 2018$

Date of acceptance: 09/12/2018

Date of publication: December 2018

\section{CASE REPORT}

\section{Background}

Laparoscopy is currently widely used for the therapeutic purposes. The minimally invasive approach has become the method of choice for the most benign disease. Creation of pneumoperitoneum is the first step of a laparoscopy. There are two methods for creating pneumoperitoneum, the closed technique, and the open technique. The VN is placed blindly into the abdomen, which increases the risk of iatrogenic injury. Reducing morbidity and mortality rates is essential to know about the major and minor complications. The complications arising from the insertion of the needle occurred in $0.23 \%$ of the $\mathrm{VN}$ accessed. ${ }^{1}$ The major complications include injury to vasculature, bladder, and bowel. Minor complications can also occur, such as wound infection, subcutaneous emphysema, and extraperitoneal insufflation. The purpose of this study was to discover the trivial complication outcome of extraperitoneal insufflation with $\mathrm{VN}$ access that had occurred during the laparoscopic surgical staging of endometrial cancer.

\section{CASE DESCRIPTION}

A 61-year-old woman, para 2 of vaginal deliveries, had previous laparoscopic GUS owing to uterine prolapse by suturing round ligaments to pull up under the anterior sheath of the rectus close to the midline abdominal wall 10 years ago (Fig. 1A). She had menorrhagia for six months after her menopausal age of 53 years. The uterine had undergone curettage, and the histopathology displayed endometrial carcinoma. The appropriate treatment was laparoscopic surgical staging. While the Veress needle was inserted and $\mathrm{CO}_{2}$ insufflation was taking place, an intraoperative complication had occurred. Two consecutive attempts were noted for the $\mathrm{VN}$ to enter the peritoneal cavity. The first attempt was a failed entry with high- 

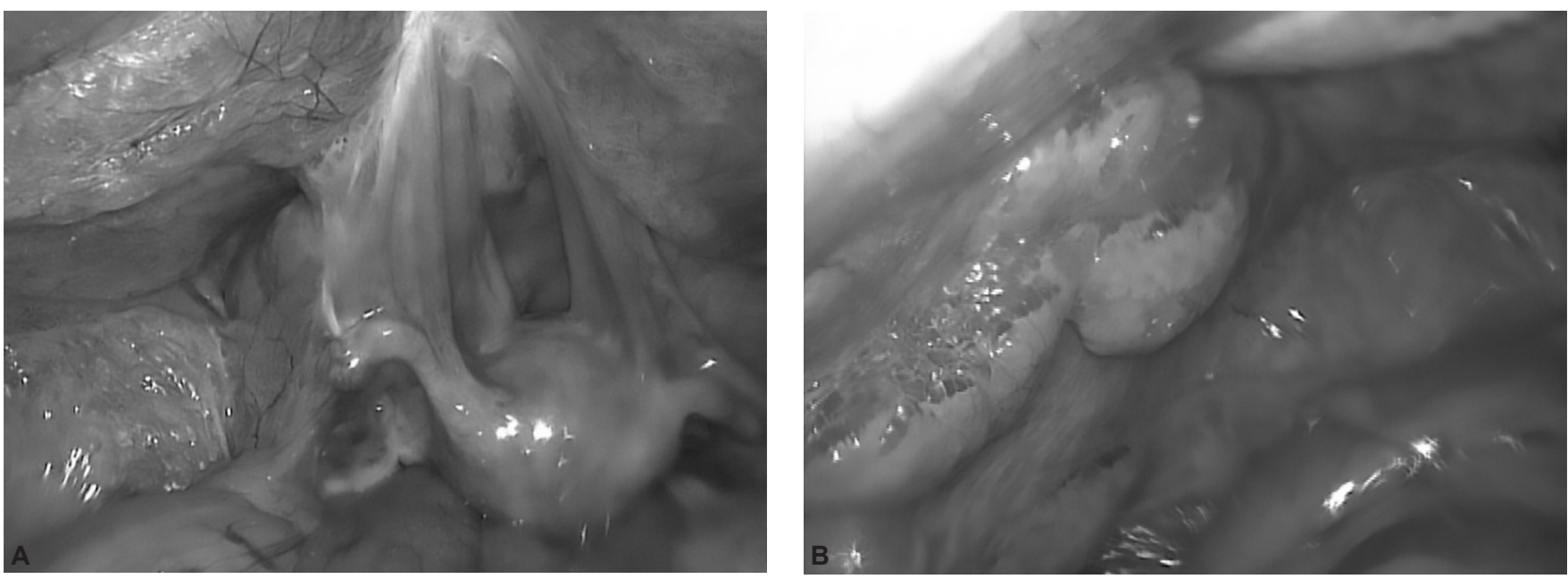

Figs. 1A and B: (A) The Gilliam uterine suspension was revealed by laparoscopy; (B) The laparoscopy showed extraperitoneal emphysema after Veress needle was insertion and $\mathrm{CO}_{2}$ was insufflation

pressure $\mathrm{CO}_{2}$ insufflation while the second attempt was able to enter the intraperitoneal cavity. The laparoscopy revealed GUS where the extraperitoneal emphysema had occurred (Fig. 1). The extraperitoneal emphysema was released with a needle gauge number 18 exteriorly during surgery.

\section{DISCUSSION}

The uterine suspension using laparoscopic visualization has been reported in England in 1976 and has become an infrequent operation on most gynecology in recent years. ${ }^{2}$ The 61-year-old female had been uterine prolapse in her age of 51 years. After the conversation with a patient, this case was designed for a laparoscopic surgical technique of GUS for a retroverted uterus with uterine prolapse that the round ligaments were sutured to the rectus fascia as shown in Figure 1A. At 10 years afterward, the patient had diagnosed with endometrial carcinoma with the histopathology of grade 1 endometrioid carcinoma after she had uterine curettage due to postmenopausal bleeding, the menopausal age was 53-year-old. The decision of surgery was a laparoscopic surgical staging. The minimally invasive can be performed to endometrial cancer safely with the utilization of the retrieval bag through a vagina to limit tissue fragmentation within a packet. ${ }^{3}$ This case was selected for the closed-entry procedure with general Veress needle technique; the two attempts were successful for VN insertion followed by the creation of pneumoperitoneum at a pressure of $15 \mathrm{~mm} \mathrm{Hg}$, the VN was removed. There is no accepted universal definition of failed closed-entry. ${ }^{4}$ Angioli defined failed entry as entry requiring more than three attempts, the extraperitoneal insufflation was seen only after VN insertion, which was a minor complication, and the incidence of extraperitoneal insufflation was 3.1\% in the VN group. ${ }^{5}$ In this case, the laparoscopy showed
Gilliam adhesion with extraperitoneal emphysema after two attempts of Veress needle insertion (Figs. 1A and B), and the emphysema was improved by using number 18 needle piercing to release its emphysema. Although the incidence of extraperitoneal insufflation and failed entry due to an open-entry method was reduced when compared to the closed-entry procedure, ${ }^{1}$ the institution is familiar to usage $\mathrm{VN}$ more than 20 years with the low incidence of complications. This report, therefore, helps to build greater awareness of extraperitoneal emphysema even though it is the minor complication. The following comprehensive staging was completed by cautious laparoscopy. Postoperative was uneventful.

\section{CONCLUSION}

The extensive extraperitoneal insufflation image shows a minor complication that is very uncommon reviewed and can thus be educational for the endoscopist.

\section{CLINICAL SIGNIFICANCE}

The VN was inserted vertically following the creation of pneumoperitoneum at a pressure of 10 to $15 \mathrm{~mm} \mathrm{Hg}$, and the VN was removed. Then, a $10 \mathrm{~mm}$ disposable shielded trocar was introduced in the pelvic cavity.

\section{ACKNOWLEDGMENT}

The study was exempt from the requirement for approval by the institutional review board of Chang Gung Memorial Hospital. The authors would like to thank the Asia-Pacific Association for gynecologic endoscopy and minimally invasive therapy (APAGE) for providing the International Fellowship Endoscopy Training Program at Chang Gung Memorial Hospital for Dr. Wasinghon. 


\section{REFERENCES}

1. Azevedo JL, Azevedo OC, Miyahira SA, Miguel GP, Becker $\mathrm{OM}$ Jr, Hypólito OH, et al. Injuries caused by Veress needle insertion for creation of pneumoperitoneum: a systematic literature review. Surg Endosc 2009;23(7):1428-1432.

2. Candy JW. Modified Gilliam uterine suspension using laparoscopic visualization. Obstet Gynecol 1976;47(2):242243.

3. Bergstrom J, Aloisi A, Armbruster S, Yen TT, Casarin A, Leitao Jr MM, et al. Minimally invasive hysterectomy surgery rates for endometrial cancer performed at national comprehensive cancer network (NCCN) centers. Gynecol Oncol 2018;148(3):480-484

4. Ahmad G, Gent D, Henderson D, O'Flynn H, Phillips K, Watson A. Laparoscopic entry techniques. Cochrane database of systematic reviews 2015; September 1. [Epub ahead of print].

5. Angioli R, Terranova C, Nardone CDC, et al. A comparison of three different entry techniques in gynecological laparoscopic surgery: a randomized prospective trial. Eur J Obstet Gynecol Reprod Biol 2013;17(2):339-342. 